\title{
NONLINEAR QUESTIONS IN CLAMPED PLATE MODELS
}

\author{
HANS-CHRISTOPH GRUNAU
}

Abstract. The linear clamped plate boundary value problem is a classical model in mechanics. The underlying differential equation is elliptic and of fourth order. The latter is a peculiar feature with respect to which this equation differs from numerous equations in physics and engineering which are of second order. Concerning the clamped plate boundary value problem, "linear questions" may be considered as well understood. This changes completely as soon as one poses the simplest "nonlinear question": What can be said about positivity preserving? Does a plate bend upwards when being pushed upwards? It is known that the answer is "no" in general. However, there are many positivity issues as e.g. "almost positivity" to be discussed.

Boundary value problems for the "Willmore equation" are nonlinear counterparts for the linear clamped plate equation. The corresponding energy functional involves curvature integrals over the unknown surface. The Willmore equation is of interest in mechanics, membrane physics and, in particular, in differential geometry. Quite far reaching results were achieved concerning closed surfaces. As for boundary value problems, by far less is known. These will be discussed in symmetric situations.

This survey article reports upon joint work with A. Dall'Acqua, K. Deckelnick (Magdeburg), S. Fröhlich (Free University of Berlin), F. Gazzola (Milan), F. Robert (Nice), Friedhelm Schieweck (Magdeburg) and G. Sweers (Cologne).

\section{Clamped plate models}

A naive measure for the bending energy of a thin elastic plate under orthogonal load $f: \Omega \rightarrow \mathbb{R}$ is given by

$$
\int_{\Omega}\left((\Delta u)^{2}-f u\right) d x
$$

Here one should think of the bounded smooth domain $\Omega \subset \mathbb{R}^{2}$ as the horizontal equilibrium shape of the plate, while $u: \Omega \rightarrow \mathbb{R}$ describes the vertical deflection from its equilibrium. $\Delta u=\sum_{i=1}^{n} \frac{\partial^{2}}{\partial x_{i}^{2}} u$ denotes its Laplacian. The corresponding Euler-Lagrange equation is the linear plate equation, which is suitable only for small deflections $u$.

This model however is not even invariant under rotating the $x_{1}, x_{2}, u$-coordinate system in $\mathbb{R}^{3}$. A more realistic measure for the bending energy without external force is given by the Willmore functional

$$
\int_{\text {graph }[u]}(H[u])^{2} d S .
$$

Here, $H$ is the mean curvature of graph $[u]$, and $d S$ denotes its surface element. The corresponding Euler-Lagrange equation is the Willmore equation.

Still, this model is rather special. For a more realistic modelling like e.g. the Helfrich functional - see e.g. [He] - as well as a lot of background information concerning variational integrals involving curvature terms we refer to the survey article by Nitsche [Nit].

Although being rather special both models give rise to a number of interesting and still largely open mathematical problems which will be outlined in the following subsections.

1991 Mathematics Subject Classification. Primary 35J40; Secondary 35J65, 35B50, 49Q10, 53C42, 34L30.

Key words and phrases. positivity, clamped plate equation, Willmore equation, Dirichlet problem, surfaces of revolution.

This survey article is based on the lecture "Some fourth order equations in mechanics and differential geometry - Old questions and some new results - " given in the Seminario Matematico Fisico di Milano on September 8, 2008.

The research was partially supported by "Deutsche Forschungsgemeinschaft." 
In Section 2 we shall explain some recent progress on positivity issues for the linear clamped plate equation while in Section 3 we present an existence result for a Dirichlet problem for the Willmore equation in a very symmetric but nevertheless interesting situation.

1.1. Linear plate equation. Throughout this article $\Omega \subset \mathbb{R}^{n}, n \geq 2$, always denotes a bounded sufficiently smooth domain. For given $f: \bar{\Omega} \rightarrow \mathbb{R}$ we look for $u: \bar{\Omega} \rightarrow \mathbb{R}$ as solution of the plate equation:

$$
\Delta^{2} u=f \text { in } \Omega .
$$

This differential equation is elliptic, of fourth order and, hence, has to be complemented by two boundary conditions. Simplifying a bit the physical modelling one may think of Navier boundary conditions

$$
u=\Delta u=0 \text { on } \partial \Omega
$$

describing a "hinged" plate. On the other hand, a horizontally clamped plate is modelled by means of Dirichlet boundary conditions:

$$
u=|\nabla u|=0 \text { on } \partial \Omega .
$$

The difference between these two most frequently considered sets of boundary conditions is illustrated in Figure 1. One may wonder whether in the simple linear plate equation (1.1),
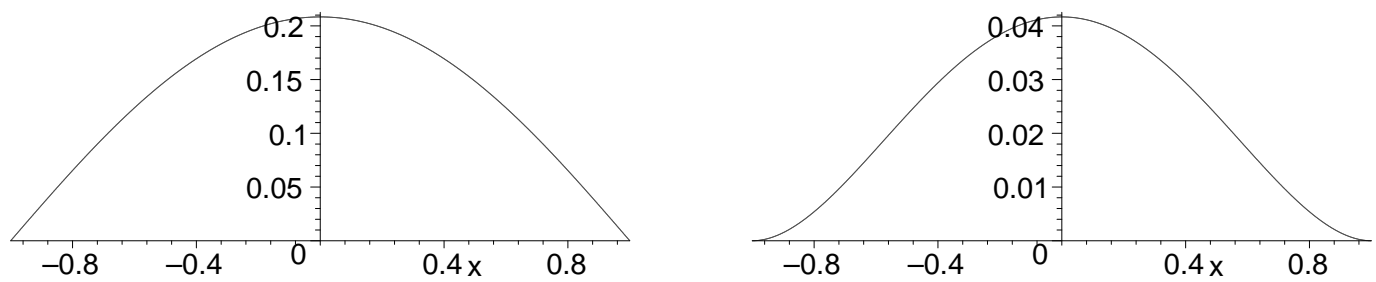

FiguRE 1 . Solution of the plate equation for $f(x) \equiv 1$ over $[-1,1]$ under Navier (left) and Dirichlet (right) boundary conditions

whether combined with Navier (1.2) or Dirichlet (1.3) boundary conditions, there still exists any open problem.

Indeed, as far as purely linear questions like existence, uniqueness and a-priori-estimates are concerned, a theory for general higher order elliptic equations under general boundary conditions was developed by Agmon, Douglis and Nirenberg [ADN] about 50 years ago.

This very satisfactory situation changes completely as soon as the simplest nonlinear question is posed:

Does the plate equation (1.1) enjoy maximum or comparison principles or, at least, a positivity preserving property?

This question is motivated by the dominating role which the maximum principle with all its variants and versions plays in the general theory of second order elliptic equations and - even more important - differential inequalities. There, it does not only permit geometric and qualitative characterisations of solutions but is also an extremely powerful technical tool.

Having a similar tool at hand also for the fourth order prototype equation (1.1) would be extremely helpful for precisely the same reasons. However, the simple example of $x \mapsto|x|^{2}$ and $x \mapsto-|x|^{2}$ shows that biharmonic functions may have any sort of local extremum without being locally constant. So, if there is any sort of positivity, it cannot be a local property but it must be a nonlocal property e.g. of the whole boundary value problem (1.1) together with either (1.2) or (1.3). Reasonably, one may ask whether the following positivity preserving property holds true:

Does a positive datum $f$ imply positivity of the solution $u$, i.e. does $f \geq 0$ always imply that $u \geq 0$ ? Do biharmonic boundary value problems enjoy comparison principles? 
For the Navier boundary value problem (1.1), (1.2) this question is easily answered in the affirmative by a twofold application of the weak maximum principle for the Laplacian. Despite this simplicity let us remark that adding lower order perturbations yields in general the same difficulties unter Navier boundary conditions as we are going to explain now for the Dirichlet problem (1.1), (1.3), see [SchJ, KaSw], cf. also the general approach in [CG].

So, in what follows we concentrate on the Dirichlet problem, i.e. on the clamped plate equation:

$$
\begin{cases}\Delta^{2} u=f & \text { in } \Omega, \\ u=|\nabla u|=0 & \text { on } \partial \Omega .\end{cases}
$$

The positivity question mentioned above may here be rephrased as follows:

Will the clamped plate bend upwards everywhere, when being pushed upwards?

This question is under investigation for more than 100 years. One reason is that by now nobody succeeded in taking advantage for our question of the seeming product structure of (1.4). Of course, one may introduce $v:=-\Delta u$ such that the differential equation becomes $-\Delta v=f$. The problem, however, is that now, $u$ is subject to two boundary conditions while for $v$, we have none.

In order to explain a bit of the historical background let us mention that one may construct a Green's function $G_{\Omega, \Delta^{2}}$ for the clamped plate equation (1.4). Then, for given datum $f$ we obtain a solution by means of the formula

$$
u(x)=\int_{\Omega} G_{\Omega, \Delta^{2}}(x, y) f(y) d y .
$$

The positivity question in any reasonable bounded domain $\Omega \subset \mathbb{R}^{n}$ is then equivalent to whether

$$
G_{\Omega, \Delta^{2}}(x, y) \geq 0 ?
$$

One may also ask for domains where strict positivity holds. Such a property would have stronger implications e.g. on positivity and in particular on simplicity of the first eigenfunction / eigenvalue.

In this direction, Boggio [Bo2] gave in 1905 a fundamental contribution. For the (unit-) ball $B=B_{1}(0) \subset \mathbb{R}^{n}$ he found an explicit formula even for any polyharmonic operator $(-\Delta)^{m}$. In the biharmonic case $m=2$, it reads

$$
G_{B, \Delta^{2}}(x, y)=\frac{1}{4 n e_{n}}|x-y|^{4-n} \int_{1}^{\sqrt{1+\frac{\left(1-|x|^{2}\right)\left(1-|y|^{2}\right)}{|x-y|^{2}}}}\left(v^{2}-1\right) v^{1-n} d v>0,
$$

where $e_{n}=\int_{B} d x$ is the $n$-dimensional volume of the unit ball, and positivity is quite immediate. A few years later, Hadamard [Ha2] commented on Boggio's work and his considerations concerning positivity of the Green's function $G_{\Omega, \Delta^{2}}$. Presumably motivated by Boggio's beautiful formula and physical intuition, he wrote quite convincingly:

Malgré l'absence de démonstration rigoureuse, l'exactitude de cette proposition ne paraît pas douteuse pour les aires convexes.

Reformulating this in terms of Green's functions, Boggio and Hadamard [Bo1, Bo2, Ha1, Ha2] conjectured:

The Green's function $G_{\Omega, \Delta^{2}}$ for the clamped plate boundary value problem in convex domains $(n=2)$ is positive.

At the same time Hadamard in [Ha2] knew that the positivity preserving property fails in annuli wuth small inner radius.

However, also the Boggio-Hadamard conjecture was disproved after 1949 by numerous authors, see e.g. [CD, D1, Ga, HJS, KKM, O, Se, ShT]. Even in arbitrarily smooth uniformly convex like mildly eccentric ellipses one has in general change of sign, see [Ga, HJS, ShT]. The failure of the positivity conjecture is illustrated in Figure 2. While the work [KKM] concerns a much deeper and more general problem, it provides in particular a counterexample to the positivity conjecture in any space dimension. For a more detailed and precise 


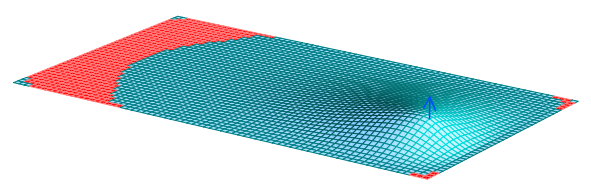

FiguRE 2. Numerical approximation: Locally acting positive force (arrow). Dark: $\{x: u(x)<0\}$. (Courtesy of Guido Sweers)

exposition of the history of this conjecture we refer to [MSh] or to the forthcoming book [GGS] and references therein.

One may wonder whether anything remains to be done concerning positivity in the clamped plate equation (1.4). When reporting these observations to physicists or engineers they usually react that they have never observed this change of sign. They propose that perhaps the model may not be perfectly suitable or that negativity is too small to be observed in reality. The latter is also supported by numerical examples where usually, the negative part is by a factor $10^{-4}$ smaller than the positive part. So, a suitable reformulation and extension of the Boggio-Hadamard conjecture towards possible applications in nonlinear problems could be:

In arbitrary domains $\Omega \subset \mathbb{R}^{n}$, the negative part of the biharmonic Green's function $G_{\Omega, \Delta^{2}}$ is small relative to the singular positive part. In the investigation of nonlinear problems, the negative part is technically disturbing but it does not give rise to any substantial additional assumption in order to have existence, regularity, etc. when compared with analogous second order problems.

The first part of this conjecture will be discussed in Section 2. The second part may by now be viewed as a kind of programme which certainly will need some time to be made working.

1.2. Willmore functional / Willmore equation. The Willmore functional of a surface $\mathcal{R} \subset \mathbb{R}^{3}$

$$
\int_{\mathcal{R}} H^{2} d S
$$

was already introduced by Poisson [Poi1] and considered by many others in the early 19th century to model thin elastic plates. The corresponding Euler-Lagrange equation is the so called Willmore equation, where we look for a surface $\mathcal{R} \subset \mathbb{R}^{3}$ solving

$$
\Delta_{\mathcal{R}} H+2 H\left(H^{2}-K\right)=0 \text { on } \mathcal{R} .
$$

Here, $\Delta_{\mathcal{R}}=\frac{1}{\sqrt{g}} \sum_{i, j} \partial_{i}\left(\sqrt{g} g^{i j} \partial_{j}\right)$ is the Laplace-Beltrami operator on the unknown surface $\mathcal{R}$. Not distinguishing between the surface and its parametrisation, $\left(g_{i j}\right)=\left(\left(\partial_{i} \mathcal{R}\right) \cdot\left(\partial_{j} \mathcal{R}\right)\right)$ is the metric tensor induced by the ambient space $\mathbb{R}^{3},\left(g^{i j}\right)$ its inverse and $g=\operatorname{det}\left(g_{i j}\right)$ its determinant. Curvatures are defined by means of the second fundamental form $L_{i j}=$ $\frac{1}{\sqrt{g}} \operatorname{det}\left(\partial_{i} \partial_{j} \mathcal{R}, \partial_{1} \mathcal{R}, \partial_{2} \mathcal{R}\right)$. The mean curvature is then given by $H=\frac{1}{2} \sum_{i, j=1}^{2} g^{i j} L_{i j}=$ $\frac{1}{2 g}\left(g_{22} L_{11}-2 g_{12} L_{12}+g_{11} L_{22}\right)$ and the Gaußian curvature by $K=\frac{\operatorname{det}\left(\left(L_{i j}\right)_{i, j=1,2}\right)}{g}$.

This differential equation (1.8) was deduced from the Willmore functional already and much before Willmore by S.D. Poisson [Poi2, p. 224]. Its invariant form is due to W. Schadow, see [Th, p. 56]. For a historical information up to 1990 we refer to the survey article by Nitsche [Nit]. However, Willmore [Wi] popularised again the mathematical study of Willmore functional and equation, while in the context of applications, physicists use a more general energy functional and speak of the Helfrich energy [He].

The Willmore equation (1.8) is of fourth order, quasilinear and non uniformly elliptic. One may observe that the surface and, hence, the Laplace-Beltrami operator are unknown and part of the problem. Fourth order equations are lacking general maximum and comparison 
principles as has become clear from the first part of this section. This means that well established methods from the theory of the second order analogue, the minimal surface equation, will fail in general. Together with the quasilinear character of (1.8) this causes great technical difficulties which so far have been overcome only in parts. The lack of uniformity in ellipticity is typical for geometric equations and occurs also in the second order minimal surface equation. On the one hand, this requires additional technical efforts but on the other hand, it also allows for specific geometric constructions and reflects some geometric invariance properties.

The lack of general comparison principles results in further drawbacks in analysing nonlinear higher order equations. A reasonable space to work in is e.g. the Sobolev space $H^{2}$. One should observe that $v \in H^{2}$ does in general not imply that $|v| \in H^{2}$. This implies that the DeGiorgi-Nash-Moser techniques are no longer applicable. Analogously, it is most difficult to define suitable auxiliary functions from solutions of higher order equations satisfying nice differential equations or inequalities. As well, there is a lack of testing functions; e.g. powers of solutions give rise to indefinite terms. The reason is that fourth order equations go together wth a set of two boundary conditions meaning that modifying an admissible testing function requires not only to match the values of the functions but also of their derivatives. All these obstructions explain why the theory of nonlinear elliptic equations of fourth order is by far less developed than of second order.

Nevertheless, concerning closed Willmore surfaces, a number of remarkable results has been achieved in the past 15 years. Combining work of Simon [Sn] and of Bauer-Kuwert [BK] one has existence of closed Willmore surfaces of any prescribed genus. Recently quite some results have been obtained concerning Willmore minimisers in fixed conformal classes [KuS4, KuS5, LP, SchM]. Riviere $[\mathrm{R}]$ proved a far reaching regularity result.

Also, local and global existence results for the Willmore flow of closed surfaces are available, see e.g. [KuS1, KuS2, KuS3, St]. On the other hand, Mayer and Simonett [MSt] gave a numerical example providing evidence that the Willmore flow may, for particular initial data, develop singularities in finite time. An analytic proof for occurence of a singularity in finite or infinite time for the same initial data was given by Blatt [Bl]. The Willmore flow for one dimensional closed curves was studied by [DKS, Pol].

This nice situation changes completely when switching to boundary value problems. Nitsche [Nit] gives some existence results which are based on perturbation arguments and, hence, require severe smallness conditions on the data which are by no means explicit. Schätzle [SchR] proved an important general result concerning existence of branched Willmore immersions in $\mathbb{S}^{n}$ with boundary which satisfy Dirichlet boundary conditions. Assuming the boundary data to obey some explicit geometrically motivated smallness condition these immersions can even be shown to be embedded. By working in $\mathbb{S}^{n}$, some compactness problems could be overcome; on the other hand, when pulling pack these immersions to $\mathbb{R}^{n}$ it cannot be excluded that they contain the point $\infty$. Moreover, in general, the existence of branch points cannot be ruled out and, due to the generality of the approach, it seems to us that no topological information about the solutions can be extracted from the existence proof. We think that it is quite interesting to identify situations where it is possible to work with a-priori-bounded minimising sequences or where solutions with additional properties like e.g. being a graph or enjoying certain symmetry properties can be found.

In order to outline possible directions of further research and to see which kind of phenomena and results concerning compact embedded solutions in $\mathbb{R}^{3}$ of fixed topological type for boundary value problems for the Willmore equation might be expected, we think that it is a good strategy to investigate first boundary value problems for (1.8) in specific symmetric situations. More precisely, we look at surfaces of revolution which are obtained by rotating a graph over the $x=x_{1}$-axis in $\mathbb{R}^{3}$ around the $x$-axis. These are described by sufficiently smooth functions

and are parametrised as follows:

$$
u:[-1,1] \rightarrow(0, \infty)
$$

$$
\mathcal{R}: \quad(x, \varphi) \mapsto(x, u(x) \cos \varphi, u(x) \sin \varphi), \quad x \in[-1,1], \quad \varphi \in[0,2 \pi] .
$$

The question we are going to address is as follows. 
Let $\alpha>0$ be given. Find an even function $u \in C^{\infty}([-1,1],(0, \infty))$ - i.e. $u(x)=u(-x)-$ such that the corresponding surface of revolution $\mathcal{R}$ minimises the Willmore energy in this class and hence solves:

$$
\left\{\begin{array}{l}
\Delta_{\mathcal{R}} H+2 H\left(H^{2}-K\right)=0 \quad \text { in }(-1,1), \\
u( \pm 1)=\alpha, \quad u^{\prime}( \pm 1)=0 .
\end{array}\right.
$$

Thanks to the symmetry assumption, the differential equation is one-dimensional which facilitates a lot the procedures to gain the requisite compactness. Due to the interplay of the principal curvatures of the unknown surface of revolution becoming apparent in the term $H^{2}-K$, the geometry is already two-dimensional and requires rather sophisticated geometric arguments. We expect these geometric constructions to give some insight in typical shapes of solutions and to outline possible strategies how to gain compactness in studying more general boundary value problems. Some of these are discussed in Sections 3.4 and 4 .

\section{Almost positivity in Biharmonic Green's FunCtions}

We now get back in some detail to the updated version and modification of the BoggioHadamard conjecture as formulated on p. 4. We shall prove below in Theorems 2.5 that in any bounded smooth domain $\Omega \subset \mathbb{R}^{n}$ the biharmonic Green's function $G_{\Omega, \Delta^{2}}$ is positive in neighbourhoods around the pole the size of which can be uniformly estimated from below irrespective of the position of the pole, provided that $n \geq 3$. As a consequence we will obtain in Theorem 2.6 much stronger estimates for $G_{\Omega, \Delta^{2}}^{-}$than for $G_{\Omega, \Delta^{2}}^{+}$and than those previously available for $\left|G_{\Omega, \Delta^{2}}\right|$. As long as the pole of the Green's function stays in compact sets such results may be obtained from the explicit form of the fundamental solution combined with elliptic estimates $[\mathrm{ADN}]$. But the difficult part is to gain uniformity when the pole approaches the boundary $\partial \Omega$ because here, the well established quantitative methods just mentioned do not yield any longer the desired information. The proof is performed by means of a rescaling and blow up technique which is sketched in [GR1] and explained in detail in [GR2]. Both are joint works with Frédéric Robert.

We start with a precise characterisation of Boggio's formula for the biharmonic Green's function in balls which serves as a basis for a perturbation theory of positivity.

2.1. Lower order perturbations and $n=2$. A perturbation theory of positivity as we shall outline it here is not just a continuous dependence on data result. In all results and arguments which we shall explain in what follows one should observe that the "Green's function" is not just a single function solving a particular Dirichlet problem. In fact, it is an infinite family of functions depending on the position of the pole. The key problem consists in gaining uniformity when the pole approaches the boundary.

The starting point is Boggio's formula (1.6) from which we deduce a precise characterisation in terms of more elementary functions which can be used more easily in what follows and explain precisely the boundary behaviour.

Proposition 2.1. (See [GS2, Proposition 2.3].)

$$
G_{B, \Delta^{2}}(x, y) \sim \begin{cases}|x-y|^{4-n} \min \left\{1, \frac{d(x)^{2} d(y)^{2}}{|x-y|^{4}}\right\}, & \text { if } n>4 ; \\ \log \left(1+\frac{d(x)^{2} d(y)^{2}}{|x-y|^{4}}\right), & \text { if } n=4 ; \\ d(x)^{2-\frac{n}{2}} d(y)^{2-\frac{n}{2}} \min \left\{1, \frac{d(x)^{\frac{n}{2}} d(y)^{\frac{n}{2}}}{|x-y|^{n}}\right\}, & \text { if } n<4 .\end{cases}
$$

Here, we denote $d(x)=\operatorname{dist}(x, \partial B)$. Moreover, $\sim$ means that up to suitable positive constants $G_{B, \Delta^{2}}$ may be estimated from below and above by the same function.

This characterisation can be used to prove so called 3-G-type theorems, i.e. that iterated Green's functions can be estimated by the Green's function itself. This allows for estimating Neumann perturbation series and one ends up with a result concerning lower order perturbations of Boggio's prototype situation. 
Proposition 2.2. (See [GS2, Theorem 5.1].) There exists $\varepsilon_{0}=\varepsilon_{0}(n)>0$ such that the Green's function $G_{B, L}$ for the operator $L u=\Delta^{2} u+\sum_{|\alpha| \leq 3} a_{\alpha} D^{\alpha} u$ under Dirichlet boundary conditions is positive provided that $\left\|a_{\alpha}\right\|_{C^{0}(\bar{B})} \leq \varepsilon_{0}$ for all $|\alpha| \leq 3$.

Restricting to two dimensions one may wonder about the interplay of conformal maps and positivity preserving for the clamped plate boundary value problem. While the Laplacian is conformally invariant the Bilaplacian is not. The only conformal maps which leave the biharmonic equation invariant are Möbius transforms (see $[\mathrm{L}]$ ). At a first glance it seems that as for positivity preserving one is caught with balls. However, taking advantage of the conformal invariance of the Laplacian one finds that the biharmonic operator is preserved as the leading principal part under conformal maps. This means that pulling back the clamped plate boundary value problem from a domain being biholomorphically close to the (unit) disk $B \subset \mathbb{R}^{2}$ gives rise to small lower order perturbations as described in Proposition 2.2. Moreover, making use of an explicit construction of biholomorphic maps by means of harmonic Green's functions (see [C]) translates closeness of domains in a differentiable sense into closeness in a biholomorphic sense. We end up with the following result saying that the positivity of the biharmonic Green's function in the two-dimensional disk $B \subset \mathbb{R}^{2}$ is preserved under small domain perturbations.

Theorem 2.3. (See [GS1, Theorem 1.5].) There exists $\varepsilon_{0}>0$ such that the following holds. We assume that $\Omega \subset \mathbb{R}^{2}$ is a $C^{4, \gamma}$-smooth domain which is $\varepsilon_{0}$-close to the disk $B$ in a $C^{4}$-sense, i.e.:

$$
\text { There exists a } C^{4} \text {-diffeomorphism } \psi: \bar{B} \rightarrow \bar{\Omega} \text { such that }\|I d-\psi\|_{C^{4}(\bar{B})} \leq \varepsilon_{0} .
$$

Then, the Green's function $G_{\Omega, \Delta^{2}}$ for $\Delta^{2}$ in $\Omega$ under Dirichlet boundary conditions is strictly positive:

$$
\forall x, y \in \Omega, \quad x \neq y: \quad G_{\Omega, \Delta^{2}}(x, y)>0 .
$$

Actually, the result in [GS1, Theorem 1.5] is more general. It covers also perturbations of $\Delta^{2}$ as leading principal part by making use of reduction to normal form and also perturbations of the polyharmonic operator $(-\Delta)^{m}$ under Dirichlet boundary conditions. Subsequently, the requisite notion of domain closeness could be reduced by Sassone [Sa] to a $C^{2, \gamma}$-sense.

The methods for proving Theorem 2.3 are strictly two-dimensional. Nevertheless, the blow-up techniques developed together with F. Robert permit to prove an analogous result in any space dimension $n \geq 3$, see Theorem 2.7 below.

2.2. Local positivity, $n \geq 3$. In what follows we shall discuss the case $n \geq 3$ and aim not only at proving an analogue of Theorem 2.3. According to the question asked on p. 4 we would like to find a precise quantitative formulation for our feeling that in any bounded regular domain, the negative part of the biharmonic Green's function is much smaller than the singular positive part.

We start with a relatively simple result concerning positivity for pairs of points being closer to each other than to the boundary. With domain dependent constants and assuming $n>4$ such a result could have been deduced from the explicit form of the biharmonic singular fundamental solution and elliptic Schauder estimates [ADN]. However, somehow remarkably, the following result can be formulated with explicit universal constants, which only depend the space dimension $n$. Generalising the result and the methods of Nehari [Ne], we have the following local positivity result, which is illustrated in Figure 3. Here and in what follows we always use the notation

$$
d(x):=\operatorname{dist}(x, \partial \Omega)
$$

Theorem 2.4. (For $n=3$, see [Ne, p. 112]. For $n \geq 4$, see [GS3, Theorem 1].) For $n \geq 3$, there exists a constant $\delta_{n}>0$ such that the following holds.

Let $\Omega \subset \mathbb{R}^{n}$ be a bounded $C^{4, \gamma}$-smooth domain. Then

$$
|x-y|<\delta_{n} \max \{d(x), d(y)\} \quad \Rightarrow \quad G_{\Omega, \Delta^{2}}(x, y)>0 .
$$


For $\delta_{n}$ one may achieve that

$$
\begin{gathered}
\delta_{3} \geq 0.53, \quad \delta_{4} \geq 0.59, \quad \delta_{n} \geq 0.6 \text { for } n \geq 5, \\
\lim _{n \rightarrow \infty} \delta_{n}=\frac{\sqrt{5}-1}{2}=0.618 \ldots
\end{gathered}
$$

For $n=2$, according to [Ne, p. 112], only a much weaker result is available.

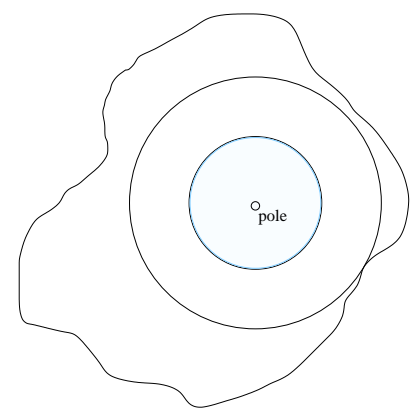

FiguRE 3. Local positivity around the singularity $(n \geq 3)$

Theorem 2.4 says that for $x \in \Omega$ one has that $G_{\Omega, \Delta^{2}}(x,$.$) is positive in B_{\delta_{n} \operatorname{dist}(x, \partial \Omega)}(x)$. The radius collapses when $x \rightarrow \partial \Omega$ and in particular, it might still be possible to find $y$ between $x$ and $\partial \Omega$ with $G_{\Omega, \Delta^{2}}(x, y)<0$. In such a case, one would expect the negative part to be of order $|x-y|^{4-n}(n>4)$ where at the same time, $|x-y|$ could become arbitrarily small. This would mean that the negative part of $G_{\Omega, \Delta^{2}}$ were of the same order of magnitude as its positive part and this is precisely what we want to exclude now. The most difficult and key step is now gaining uniformity in the local positivity result when the pole approaches the boundary. This means that we have to replace $B_{\delta_{n} \operatorname{dist}(x, \partial \Omega)}(x)$ as a region of positivity by a ball of uniform radius around any $x$ intersected with $\Omega$, see Figure 4 .

Theorem 2.5. (See [GR1, Theorem 1.1].) Let $\Omega \subset \mathbb{R}^{n}, n \geq 3$, be a bounded $C^{4, \gamma}$-smooth domain. Then, there exists a constant $\delta(\Omega)>0$ such that

$$
x, y \in \Omega, \quad x \neq y, \quad|x-y|<\delta \Rightarrow G_{\Omega, \Delta^{2}}(x, y)>0 .
$$
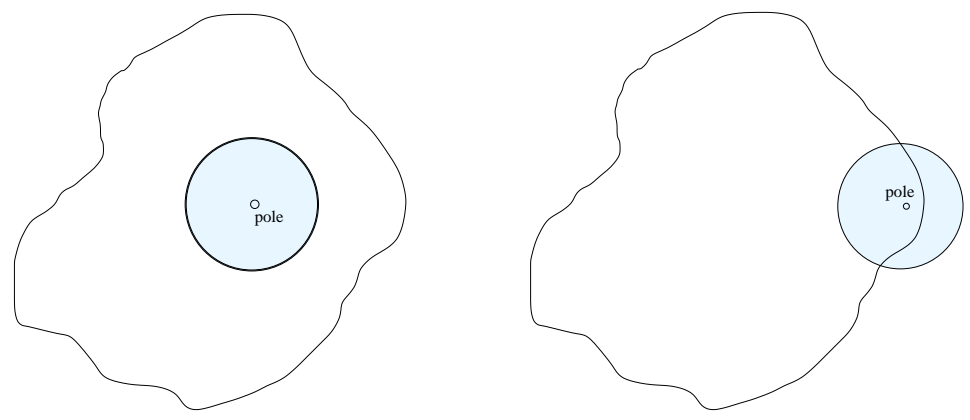

FIGURE 4. Uniform positivity around the pole, $n \geq 3$.

Since Theorem 2.4 is used in the proof and not available for $n=2$ we are likewise not in the position to extend Theorem 2.5 to $n \geq 2$.

Proof. We sketch the main ideas for the generic case $n>4$. The cases $n=3,4$ are special and technically more involved. For details one may see [GR2], where also more general questions are studied.

We assume by contradiction that we find sequences $x_{k}, \tilde{y}_{k} \in \Omega, x_{k} \neq \tilde{y}_{k}$ which satisfy $G_{\Omega, \Delta^{2}}\left(x_{k}, \tilde{y}_{k}\right) \leq 0$ and $\lim _{k \rightarrow \infty}\left|x_{k}-\tilde{y}_{k}\right|=0$. In view on the smoothness of $\partial \Omega$ we find 
a further sequence $y_{k} \in \Omega$ with $x_{k} \neq y_{k}, G_{\Omega, \Delta^{2}}\left(x_{k}, y_{k}\right)=0$ and $\lim _{k \rightarrow \infty}\left|x_{k}-y_{k}\right|=0$. Theorem 2.4 yields that

$$
\left|x_{k}-y_{k}\right| \geq \delta_{n} \max \left\{d\left(x_{k}\right), d\left(y_{k}\right)\right\},
$$

where $\delta_{n}>0$ may be taken from there. After passing to a subsequence we find a limit $x_{\infty}=\lim _{k \rightarrow \infty} x_{k}=\lim _{k \rightarrow \infty} y_{k} \in \partial \Omega$. By means of a rotation and translation we may achieve that $x_{\infty}=0$ and that the first unit vector is the exterior normal in 0 at $\partial \Omega$. By means of a blow up or rescaling procedure we shall deduce properties of the biharmonic Green's function in a half space as a limit domain which will contradict the explicit formula due to Boggio.

To this end, for $k$ large enough, we may define $\tilde{x}_{k} \in \partial \Omega$ as the closest boundary point to $x_{k}$. We introduce the rescaled biharmonic Green's functions

$$
G_{k}(\xi, \eta):=\left|x_{k}-y_{k}\right|^{n-4} G_{\Omega, \Delta^{2}}\left(\tilde{x}_{k}+\left|x_{k}-y_{k}\right| \xi, \tilde{x}_{k}+\left|x_{k}-y_{k}\right| \eta\right)
$$

for

$$
\xi, \eta \in \Omega_{k}:=\frac{1}{\left|x_{k}-y_{k}\right|}\left(-\tilde{x}_{k}+\Omega\right) .
$$

Since $\tilde{x}_{k} \rightarrow 0$ and so, the exterior unit normal at $\partial \Omega$ converges to the first unit vector we conclude that

$$
\Omega_{k} \rightarrow \mathcal{H}:=\left\{x: x_{1}<0\right\} \text { locally uniformly for } k \rightarrow \infty .
$$

In order to gain compactness for $\left(G_{k}\right)_{k \in \mathbb{N}}$, we refer to the following estimates which were proved first by Krasovskiı [Kr1, Kr2] and where in the particular case of the Bilaplacian the smoothness assumption on $\partial \Omega$ were relaxed in [GR2, Theorem 4]:

$$
\left|G_{\Omega, \Delta^{2}}(x, y)\right| \leq C_{0}|x-y|^{4-n} .
$$

The constant $C_{0}$ may be chosen uniformly for smooth families of domains. Thanks to these estimates we see that for any $\xi, \eta \in \mathcal{H}$ and $k$ large enough

$$
\left|G_{k}(\xi, \eta)\right| \leq C_{0}|\xi-\eta|^{4-n} \text { uniformly in } k \text {. }
$$

Combining these pointwise bounds, elliptic estimates [ADN], biharmonic reflection principles [D2] and biharmonic Liouville theorems [Nic], one may prove that locally uniformly in $\mathcal{H}$

$$
G_{k}(\xi, \eta) \rightarrow G_{\mathcal{H}, \Delta^{2}}(\xi, \eta)=\frac{1}{4 n e_{n}}|\xi-\eta|^{4-n} \int_{1}^{\left|\xi^{*}-\eta\right| /|\xi-\eta|}\left(v^{2}-1\right) v^{1-n} d v
$$

where $\xi^{*}=\left(-\xi_{1}, \xi_{2}, \ldots, \xi_{n}\right)$. For details we refer to [GR2, Lemma 7]. The identity for $G_{\mathcal{H}, \Delta^{2}}$ is due to Boggio's formula (1.6) transformed to the half space $\mathcal{H}$ by means of a suitable Möbius transform (or verified directly). The assumption that $G_{\Omega, \Delta^{2}}\left(x_{k}, y_{k}\right)=0$ gives

$$
G_{k}\left(\xi_{k}, \eta_{k}\right)=0
$$

where

$$
\begin{gathered}
\xi_{k}=\frac{1}{\left|x_{k}-y_{k}\right|}\left(x_{k}-\tilde{x}_{k}\right), \quad \eta_{k}=\frac{1}{\left|x_{k}-y_{k}\right|}\left(y_{k}-\tilde{x}_{k}\right), \\
\left|\xi_{k}\right|=\frac{d\left(x_{k}\right)}{\left|x_{k}-y_{k}\right|} \leq \frac{1}{\delta_{n}}, \quad\left|\xi_{k}-\eta_{k}\right|=1 .
\end{gathered}
$$

After passing to a further subsequence we find $\xi, \eta \in \overline{\mathcal{H}}$ with $\xi=\lim _{k \rightarrow \infty} \xi_{k}, \eta=\lim _{k \rightarrow \infty} \eta_{k}$. Working in local coordinate charts, using Taylor's formula, and exploiting the convergence properties of $G_{k}$ we see that $G_{k}\left(\xi_{k}, \eta_{k}\right)=0$ yields one of the three possibilities:

(1) $\xi \in \mathcal{H}, \eta \in \mathcal{H}, G_{\mathcal{H}, \Delta^{2}}(\xi, \eta)=0$;

(2) $\xi \in \mathcal{H}, \eta \in \partial \mathcal{H}, \Delta_{\eta} G_{\mathcal{H}, \Delta^{2}}(\xi, \eta)=0$, or vice versa;

(3) $\xi \in \partial \mathcal{H}, \eta \in \partial \mathcal{H}, \Delta_{\xi} \Delta_{\eta} G_{\mathcal{H}, \Delta^{2}}(\xi, \eta)=0$.

We have achieved a contradiction because according to Boggio's formula, none of these possibilities may indeed occur. 
2.3. Bounds for the negative part $n \geq 3$. In the previous section we have proved that close to the singularity, no sign change of the biharmonic Green's function $G_{\Omega, \Delta^{2}}$ may occur, irrespective of how close the singularity is to the boundary.

This observation together with estimates due to Krasovskiı [Kr1, Kr2] and refined by Dall'Acqua and Sweers [DS] can now be turned into a partial answer to the question posed on p. 4. We obtain a much stronger estimate for the negative part $G_{\Omega, \Delta^{2}}^{-}$than for the singular positive part.

Theorem 2.6. (See [DMS], [DS], [GR1, Theorem 1.1].) Let $\Omega \subset \mathbb{R}^{n}, n \geq 2$, be a bounded $C^{4, \gamma}$-smooth domain. We abbreviate $G(x, y):=G_{\Omega, \Delta^{2}}(x, y)$. Then, there exists a constant $C=C(\Omega)$ such that for all $x, y \in \Omega, x \neq y$ one has:

$$
-C d(x)^{2} d(y)^{2} \leq G(x, y) \leq C \begin{cases}|x-y|^{4-n} \min \left\{1, \frac{d(x)^{2} d(y)^{2}}{|x-y|^{4}}\right\}, & \text { if } n>4 \\ \log \left(1+\frac{d(x)^{2} d(y)^{2}}{|x-y|^{4}}\right), & \text { if } n=4 \\ (d(x) d(y))^{2-\frac{n}{2}} \min \left\{1, \frac{d(x)^{\frac{n}{2}} d(y)^{\frac{n}{2}}}{|x-y|^{n}}\right\}, & \text { if } n<4 .\end{cases}
$$

For $x, y$ closer to the boundary than to each other, the bound from below is by a factor $|x-y|^{n}$ smaller than the bound from above.

Garabedian [Ga] proved change of sign for $G_{\Omega, \Delta^{2}}$ with $\Omega \subset \mathbb{R}^{2}$ a mildly eccentric ellipse by finding opposite boundary points $x_{0}, y_{0}$ with $\Delta_{x} \Delta_{y} G_{\Omega, \Delta^{2}}\left(x_{0}, y_{0}\right)<0$. This shows that qualitatively, the estimate from below cannot be further improved.

Proposition 2.1 shows that also the bound from above is qualitatively optimal.

Proof. Basing upon Krasovski1's work [Kr1, Kr2], it was proved in [DS] that $|G(x, y)|$ can be estimated from above by the term on the right hand side in the statement of the theorem. Originally, due to a much more general setting, these results require more smoothness on $\partial \Omega$. However, as can be seen from [GR2, Theorem 4] and [GGS, Chapter 3], $C^{4, \gamma_{\text {- }}}$ smoothness is enough. If $n \geq 3$, the estimate from below follows immediately from the previous Theorem 2.5. If $n=2$, these techniques don't seem to work any longer, but the statement was proved taking advantage of conformal maps by Dall'Acqua, Meister and Sweers in [DMS]. Originally, they had to impose much stronger smoothness assumptions on $\partial \Omega$. However, since the Krasovskiu-type estimates are for our special problem available also in $C^{4, \gamma}$-smooth domains, this smoothness is sufficient for the estimate of the Green's function from below ([GGS, Chapter 3], [DA]).

It remains as an interesting project to prove smallness of the negative part also in different norms or by finding explicit (small) values for the constant in the estimate from below. Since the underlying Theorem 2.5 was proved by contradiction, here this constant cannot be calculated explicitly, even not in principle.

2.4. Domain perturbations also in $n \geq 3$. Theorem 2.5 can be proved also in smooth families of domains while the strategy of proof remains exactly the same. As a consequence one can describe how within a smooth family of domains, the transition from positivity to a possible change of sign may occur. In particular, if $n \geq 3$, this cannot happen via the singularity but similarly as described at the end of the proof of Theorem 2.5. Boggio's formula shows that none of these possibilities may occur in a ball. Hence, one has the following higher dimensional analogue of the perturbation result Theorem 2.3.

Theorem 2.7. (See [GR2, Theorem 2].) Let $n \geq 3$. Then, there exists $\varepsilon_{0}=\varepsilon_{0}(n)>0$ such that the following holds.

We assume that $\Omega \subset \mathbb{R}^{n}$ is a $C^{4, \gamma}$-smooth domain which is $\varepsilon_{0}$-close to the ball $B$ in the $C^{4, \gamma}$-sense, i.e. there exists a $C^{4, \gamma}$-diffeomorphism $\psi: \bar{B} \rightarrow \bar{\Omega}$ such that $\|I d-\psi\|_{C^{4, \gamma}(\bar{B})} \leq$ $\varepsilon_{0}$.

Then, the Green's function $G_{\Omega, \Delta^{2}}$ for $\Delta^{2}$ in $\Omega$ under Dirichlet boundary conditions is strictly positive:

$$
\forall x, y \in \Omega, \quad x \neq y: \quad G_{\Omega, \Delta^{2}}(x, y)>0 .
$$


Analogolously as for Theorem 2.3 one may wonder whether the notion of smoothness could be relaxed to a $C^{2, \gamma}$-sense. We expect this to be true but also the proof to be quite technical.

2.5. Perspectives. So far, Theorem 2.6 explains e.g. for the linear clamped plate boundary value problem that the deviation from having a comparison principle or a positivity preserving property is not too large. On the whole, the response of the solution has the same direction as the datum.

In view of possible applications to nonlinear problems, one would need a generalisation of Theorem 2.6 for classes of operators with lower order coefficients. As long as these coefficients are bounded, one has such uniform estimates. For unbounded families of coefficients, uniformity breaks down which limits the applicability of Theorem 2.6 to nonlinear equations. For such purposes one should think of developing this result for different norms of the fundamental solution (e.g. $L^{1}$ ) and try to find "small" constants for the norms of the negative parts of the Green's functions.

\section{The Dirichlet Problem for Willmore surfaces of ReVolution}

In order to get a first insight which sort of existence phenomena may be expected for classical bounded Willmore surfaces under Dirichlet boundary conditions we study a particularly symmetric situation which nevertheless displays already some of the typical two-dimensional geometric features. We are going to construct Willmore surfaces of revolution :

$$
\mathcal{R}:(x, \varphi) \mapsto(x, u(x) \cos \varphi, u(x) \sin \varphi), x \in[-1,1], \varphi \in[0,2 \pi],
$$

with sufficiently smooth $u:[-1,1] \rightarrow(0, \infty)$, subject to Dirichlet boundary conditions. One may think of cylindrical elastic surfaces being clamped at their ends. We explain in some detail the result concerning horizontal clamping, i.e. $u^{\prime}( \pm 1)=0$, because already here, some basic geometric features show up. In Theorem 3.6 below, we mention a result concerning general symmetric sets of boundary data.

Theorem 3.1. (See [DDG, Theorem 1.1] and [DFGS, Theorem 3.18, Lemma 3.20].) For each $\alpha>0$ there exists $u \in C^{\infty}([-1,1],(0, \infty))$ such that the corresponding surface of revolution solves the following Dirichlet problem:

$$
\left\{\begin{array}{l}
\Delta_{\mathcal{R}} H+2 H\left(H^{2}-K\right)=0 \quad \text { in }(-1,1) \\
u( \pm 1)=\alpha, \quad u^{\prime}( \pm 1)=0
\end{array}\right.
$$

The solution $u$ we construct is even and satisfies for all $x \in(0,1): u^{\prime}(x)<0,0<x+$ $u(x) u^{\prime}(x)$. Moreover, we have for all $x \in[-1,1]$ the following estimates:

$$
\alpha \leq u(x) \leq \sqrt{\alpha^{2}+1-x^{2}}, \quad\left|u^{\prime}(x)\right| \leq \frac{|x|}{\alpha} .
$$

This result will be proved by minimising the Willmore functional in the class of all positive even $C^{1,1}$-functions on $[-1,1]$ satisfying the boundary conditions above. Since the derivation of the corresponding Euler-Lagrange-equation is well established we refer for this issue e.g. to [DDG, DG, Nit, Poi1, Poi2, Th].

So, in what follows we concentrate on minimising the Willmore functional. For this purpose we need a formula for the mean curvature of the surface of revolution $\mathcal{R}$ given by $u$ :

$$
H=-\frac{u^{\prime \prime}(x)}{2\left(1+u^{\prime}(x)^{2}\right)^{3 / 2}}+\frac{1}{2 u(x) \sqrt{1+u^{\prime}(x)^{2}}} .
$$

Hence the corresponding Willmore functional is given by

$$
\begin{aligned}
& W(\mathcal{R})= \int_{\mathcal{R}} H^{2} d S \\
&=\frac{\pi}{2} \int_{-1}^{1}\left(-\frac{u^{\prime \prime}(x)}{\left(1+u^{\prime}(x)^{2}\right)^{3 / 2}}+\frac{1}{u(x) \sqrt{1+u^{\prime}(x)^{2}}}\right)^{2} \\
& \cdot u(x) \sqrt{1+u^{\prime}(x)^{2}} d x .
\end{aligned}
$$


Although $u$ is a function just in one variable, this functional looks most discouraging, at least from an analytic point of view. The two terms in $H$ compete with each other: The first penalises large curvatures of $u$ while the second one has a preference for large $u$ and almost vertical curves. Geometrically, the functional prefers minimal surfaces, i.e. catenoids. However, there is not an obvious way to construct good admissible functions just with the help of these. In this direction, hyperbolic geometry will offer a different point of view.

3.1. Hyperbolic geometry. It has been known since the contributions by Bryant and Griffiths $[\mathrm{Br}, \mathrm{BG}]$ that Willmore surfaces of revolution may be equivalently considered as elastic curves in the hyperbolic half plane. This observation was also made by Pinkall (and communicated e.g. in $[\mathrm{HP}])$. The differential equation for the hyperbolic curvature takes a relatively simple shape which was used by Langer and Singer [LS1, LS2] to classify it in terms of elliptic functions of the hyperbolic arclength of the unknown curves. In spite of this relatively explicit situation we did not see a way to integrate these equations in order to solve directly the Dirichlet problem in question.

Nevertheless, we take great advantage of this reformulation of the Willmore problem in the hyperbolic half plane since it will allow for geometric constructions which ensure sufficient compactness on suitable minimising sequences. These constructions explain to a good extent the shape of minimisers and are useful also in more general situations like e.g. in the general Dirichlet problem (3.3).

In what follows we are going to explain only the main ideas; for details we refer to [DDG, DFGS].

The hyperbolic half plane $\mathbb{R}_{+}^{2}:=\{(x, y): y>0\}$ is equipped with the metric

$$
d s^{2}=\frac{1}{y^{2}}\left(d x^{2}+d y^{2}\right)
$$

The hyperbolic curvature of $[-1,1] \ni x \mapsto(x, u(x)) \in \mathbb{R}_{+}^{2}$ is then

$$
\kappa(x)=\frac{u(x) u^{\prime \prime}(x)}{\left(1+u^{\prime}(x)^{2}\right)^{3 / 2}}+\frac{1}{\sqrt{1+u^{\prime}(x)^{2}}} .
$$

This formula is decuced by differentiating covariantly the tangent field of the graph of $u$. The curvature $\kappa$ is defined as the proportionality factor of this derivative and the unit normal. However, no geometric knowledge is required in what follows except concerning hyperbolic line integrals and that the (hyperbolic) geodesics - i.e. curves with $\kappa(x) \equiv 0$ - are precisely subsets of circles centered on the $x$-axis or of vertical straight lines. See Figure 5.

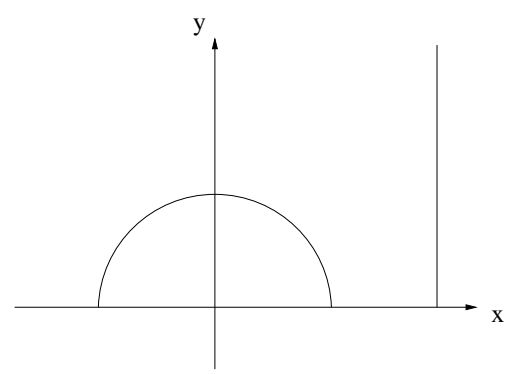

Figure 5. Hyperbolic geodesics

We introduce the hyperbolic Willmore functional as the elastic energy of the graph of $u$ as a curve in the hyperbolic half plane. It turns out that - up to a factor and a boundary 
term - this functional coincides with the original Willmore functional.

$$
\begin{aligned}
\hat{W}(u):= & \int_{-1}^{1} \kappa(x)^{2} d s(x)=\int_{-1}^{1} \kappa(x)^{2} \frac{\sqrt{1+u^{\prime}(x)^{2}}}{u(x)} d x \\
= & \int_{-1}^{1}\left(\frac{u^{\prime \prime}}{\left(1+u^{\prime 2}\right)^{3 / 2}}-\frac{1}{u \sqrt{1+u^{\prime 2}}}\right)^{2} u \sqrt{1+u^{\prime 2}} d x \\
& +4 \int_{-1}^{1} \frac{u^{\prime \prime}(x)}{\left(1+u^{\prime}(x)^{2}\right)^{3 / 2}} d x \\
= & \frac{2}{\pi} \int_{\mathcal{R}} H^{2} d S+4\left[\frac{u^{\prime}(x)}{\sqrt{1+u^{\prime}(x)^{2}}}\right]_{-1}^{1}, \\
W(\mathcal{R})= & \frac{\pi}{2} \hat{W}(u)-2 \pi\left[\frac{u^{\prime}(x)}{\sqrt{1+u^{\prime}(x)^{2}}}\right]_{-1}^{1} .
\end{aligned}
$$

This means that as long as the boundary angles are kept fixed one may work with $W$ as well as with $\hat{W}$, depending on which appears to be more suitable or convenient. Since here, we are working with the special Dirichlet boundary conditions $u^{\prime}( \pm 1)=0$, for these functions we have

$$
\hat{W}(u)=\frac{2}{\pi} W(\mathcal{R}) .
$$

So, in what follows, for given $\alpha \in(0, \infty)$ we shall minimise $\hat{W}$ in the following class of admissible functions:

$$
\begin{aligned}
N_{\alpha} & :=N_{\alpha}([-1,1]) \\
& :=\left\{v \in C^{1,1}([-1,1]), u \text { is even and positive, } v(1)=\alpha, v^{\prime}(1)=0\right\},
\end{aligned}
$$

We define the infimum we want to achieve:

$$
M_{\alpha}:=\inf \left\{\hat{W}(v): v \in N_{\alpha}\right\} .
$$

3.2. Properties of suitable minimising sequences. We explain the most important basic constructions how to achieve suitable minimising properties. They will satisfy strong enough a-priori-estimates ensuring compactness and allowing to construct an optimal element in $N_{\alpha}$ for the minimal Willmore energy $M_{\alpha}$. More precisely, one first obtains a minimiser in the $H^{2}$-closure of $N_{\alpha}$ which is then shown to be smooth. With the same techniques one can also prove monotonicity of the minimal Willmore energy $M_{\alpha}$ with respect to $\alpha$.

In what follows we choose some $\alpha>0$ and keep it fixed.

In a first step we explain how to shorten a function thereby decreasing its hyperbolic Willmore energy. In order to keep the exposition simple, here we explain this procedure only for functions in $v \in N_{\alpha}$ with $v^{\prime}(x)<0$ on $(0,1)$.

Lemma 3.2. (See [DDG, Lemma 3.3].) Assume $v \in N_{\alpha}$ and that $v^{\prime}(x)<0$ for all $x \in(0,1)$. Then, for all $\rho \in(0,1]$, there exists $v_{\rho} \in N_{\alpha}([-\rho, \rho])$ such that $v_{\rho}^{\prime}(x)<0$ for all $x \in(0, \rho)$ and

$$
\int_{-\rho}^{\rho} \kappa\left[v_{\rho}\right]^{2} d s\left[v_{\rho}\right] \leq \hat{W}(v)
$$

Proof. For $r \in[0,1]$ we consider the geodesic circle tangent to the graph of $u$ in $(r, u(r))$ and centered in the point $\left(r+u^{\prime}(r) u(r), 0\right)$. We build a new function $v$ with lower Willmore energy. On $\left[r+u^{\prime}(r) u(r), r\right)$ we take the previously mentioned geodesic arc not contributing at all to the hyperbolic Willmore energy, while on $[r, 1]$ we keep $u$. We shift this function such that $r+u^{\prime}(r) u(r)$ is moved to the origin and extending it by symmetry, we obtain a symmetric $C^{1,1}$-function with lower Willmore energy. Depending on the parameter $r$, this new function is defined on the interval $\left[-1+r+u^{\prime}(r) u(r), 1-r-u^{\prime}(r) u(r)\right]$. By the mean value theorem we find a suitable $r$ such that $1-r-u^{\prime}(r) u(r)=\rho$. The procedure is illustrated in Figure 6. 


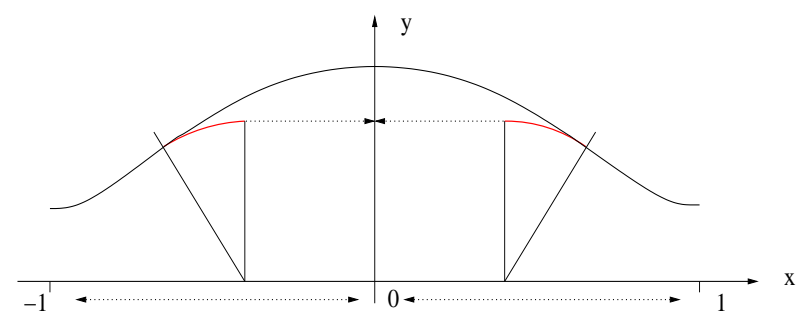

Figure 6 . How to shorten a function in $N_{\alpha}$ while decreasing its hyperbolic Willmore energy.

In [DDG, Lemma 3.5] we explain how to shorten a general function in $N_{\alpha}$ with at most finitely many critical points while decreasing its Willmore energy. Not only in this respect it is most important to know that it is most suitable for functions in $N_{\alpha}$ to decrease on $[0,1]$. We emphasise that obvious attempts like reflecting graphs about horizontal lines do not yield any conclusion: The two terms in $\kappa^{2} d s$ act against each other and it is not obvious in which term the change is larger.

Instead, a refined geometric construction is needed to get the desired result. In the following lemma, we explain how to change the bad situation displayed in Figure 7 into a nice one.

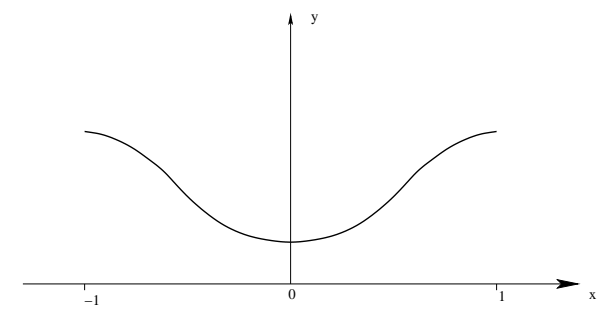

Figure 7. Too high Willmore energy.

Lemma 3.3. (See [DDG, Lemma 3.4].) Assume $u \in N_{\alpha}$ and that $u^{\prime}(x)>0$ for all $x \in(0,1)$. Then, there exists $v \in N_{\alpha}$ such that $v^{\prime}(x)<0$ for all $x \in(0,1)$ and

$$
\hat{W}(v) \leq \hat{W}(u) .
$$

Proof. In a first step we interchange the left and right part of $u$. Thanks to the boundary condition $u^{\prime}( \pm 1)=0$ we obtain a function $v_{1} \in N_{u(0)}$ with $v_{1}^{\prime}<0$ on $(0,1)$. We apply the shortening procedure of Lemma 3.2 to $v_{1}$ with $\rho=u(0) / u(1)=v_{1}(1) / v_{1}(0)$. We obtain $v_{2} \in$ $N_{u(0)}([-u(0) / u(1), u(0) / u(1)])$ with $v_{2}^{\prime}<0$ on $(0, u(0) / u(1))$ and lower Willmore energy than $u$. We come up with the desired $v$ by rescaling and define $v(x)=u(1) / u(0) v_{2}(u(0) / u(1) x)$. We emphasise that the Willmore functional $\hat{W}$ is invariant under Möbius transforms of $\mathbb{R}^{3}$. In particular, $\hat{W}$ is scaling invariant. This procedure is illustrated in Figure 8.

In [DDG, Theorem 3.8], we explain the procedure how to change a general function in $N_{\alpha}$ with at most finitely many critical points into a function in $N_{\alpha}$ with lower Willmore energy and decreasing on $[0,1]$. This requires a number of iterative applications of Lemmas 3.2 and 3.3 .

This means that on elements $v \in N_{\alpha}$ of a suitable minimising sequence we may assume that $v^{\prime} \leq 0$ on $[0,1]$. Finally we explain that it is suitable for $v$ to satisfy also the condition $0 \leq x+v(x) v^{\prime}(x)$ on $[0,1]$. For $x=1$, one has $1+v(1) v^{\prime}(1)=1>0$. Assuming that $1+v v^{\prime}<0$ somewhere in $(0,1)$, one finds a largest point $x_{0}$ with $0=x_{0}+v\left(x_{0}\right) v^{\prime}\left(x_{0}\right)$. This means that the geodesic circle centered at the origin and going through $\left(x_{0}, v\left(x_{0}\right)\right)$ is tangential to the graph of $v$. So, on $\left[-x_{0}, x_{0}\right]$ one may replace the original function $v$ by this geodesic arc thereby decreasing the Willmore energy of $v$, see Figure 9. 


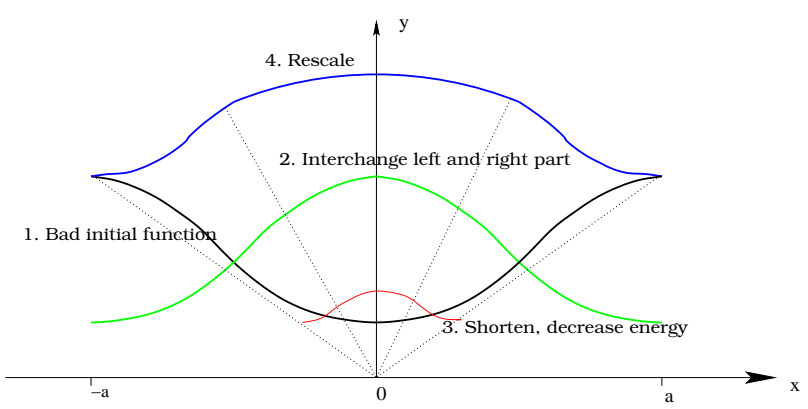

Figure 8. How to change a profile bending downwards into a profile bending upwards with lower Willmore energy.

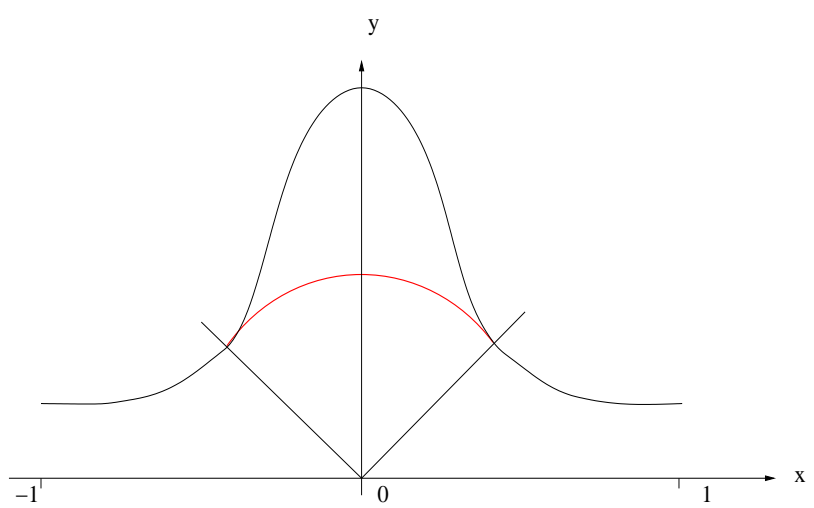

Figure 9. Elements $v$ of suitable minimising sequences obey $0 \leq x+$ $v(x) v^{\prime}(x)$ on $[0,1]$.

With these techniques one may prove the following two results, which are the key steps in proving existence of Willmore surfaces of revolution satisfying Dirichlet boundary conditions.

Theorem 3.4. (See [DDG, Theorem 3.7].) The optimal Willmore energies $(0, \infty) \ni \alpha \mapsto$ $M_{\alpha} \in[0, \infty)$ are decreasing in $\alpha$.

For fixed $\alpha$, we consider a minimising sequence

$$
\left(v_{\alpha, k}\right)_{k \in \mathbb{N}} \subset N_{\alpha}
$$

such that each $v_{\alpha, k}$ has only finitely many critical points.

Theorem 3.5. (See [DDG, Theorem 3.8 and Condition (3.6)].) One may achieve a minimising sequence $\left(v_{\alpha, k}\right)_{k \in \mathbb{N}}$ satisfying

$$
\forall x \in[0,1]: v_{\alpha, k}^{\prime}(x) \leq 0, \quad x+v_{\alpha, k}(x) v_{\alpha, k}^{\prime}(x) \geq 0 .
$$

Hence, one has for all $x \in[-1,1]$ :

$$
\alpha \leq v_{\alpha, k}(x) \leq \sqrt{1+\alpha^{2}-x^{2}}, \quad\left|v_{\alpha, k}(x)\right| \leq \frac{|x|}{\alpha} .
$$

These a priori estimates on suitably modified minimising sequences are strong enough to yield strong $C^{1}$ - and weak $H^{2}$-compactness. Then, it is straightforward to prove existence of a minimum $u$ in the $H^{2}$-closure of $N_{\alpha}$ for $M_{\alpha}$. For the proof that $u$ is indeed a $C^{\infty}$-smooth solution of (3.1) we refer to [DDG, Step 2 of the proof of Theorem 3.9].

We remark that it is proved in [DFGS] that the inequalities for our solution $u$ in Theorem 3.1 are all strict on the open intervals. Analogously, also $(0, \infty) \ni \alpha \mapsto M_{\alpha} \in[0, \infty)$ is strictly decreasing. 
3.3. Numerically calculated solutions. We display a couple of numerically calculated solutions, see Figures 10 and 11. These calculations are based on the corresponding parabolic flow equation and were performed by Friedhelm Schieweck using $C^{1}$-finite elements, see [DFGS, Chapter 7].
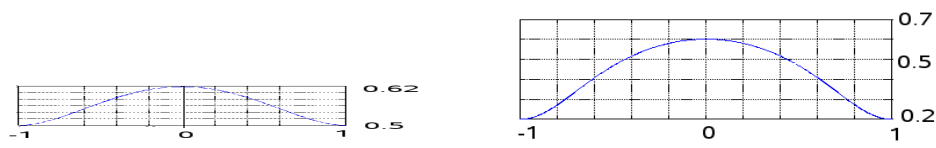

Figure 10. $\alpha=0.5$ (left) and $\alpha=0.2$ (right). (Courtesy of Friedhelm Schieweck)
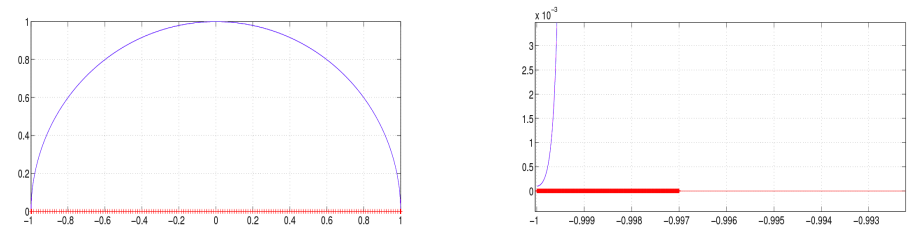

Figure 11. $\alpha=0.0001$ (Courtesy of Friedhelm Schieweck)

3.4. General Dirichlet boundary conditions. Concerning general symmetric sets of Dirichlet boundary data one has the following existence result.

Theorem 3.6. (See [DFGS, Theorem 1.1].) For each $\alpha>0$ and each $\beta \in \mathbb{R}$ there exists $u \in C^{\infty}([-1,1],(0, \infty))$ such that the corresponding surface of revolution solves the Dirichlet problem for the Willmore equation:

$$
\left\{\begin{array}{l}
\Delta_{\mathcal{R}} H+2 H\left(H^{2}-K\right)=0 \quad \text { in }(-1,1), \\
u( \pm 1)=\alpha, \quad \mp u^{\prime}( \pm 1)=\beta .
\end{array}\right.
$$

The geometric constructions developed in the proof of Theorem 3.1 also play a role here. However, in particular for $\beta<0$, the proof is much more involved. Here, also the catenoids as minimal surface solutions to the Willmore equation come into play. It is enough to consider comparison functions $v$ where for some $x_{0} \in[0,1)$ one has $v^{\prime}<0$ on $\left(0, x_{0}\right)-$ possibly, this interval may also be void - and $v^{\prime}>0$ on $\left(x_{0}, 1\right)$. Roughly speaking, catenoids are crucial on $\left[-1,-x_{0}\right] \cup\left[x_{0}, 1\right]$ while the ideas of Theorem 3.1 apply on $\left[-x_{0}, x_{0}\right]$. For general boundary data, the technical difficulties consist in finding an appropriate balance in suitable minimising sequences between these two prototype optima - catenoids and spheres. A numerically calculated Willmore surface of revolution for the particular Dirichlet data $\alpha=0.5, \beta=-10$ is displayed in Figure 12 .

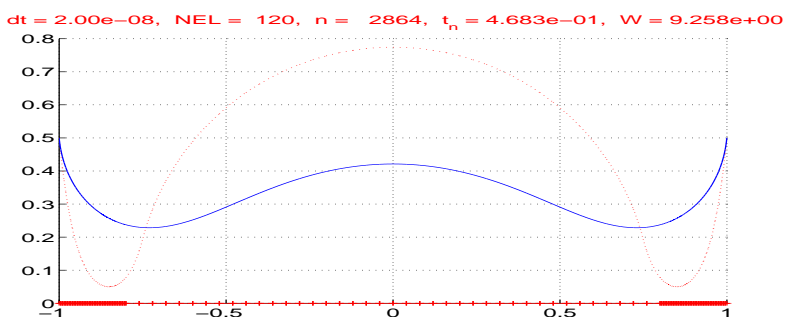

Figure 12. Numerically calculated Willmore surface, $\alpha=0.5, \beta=-10$. Dotted line: Initial datum used for the flow method. (Courtesy of Friedhelm Schieweck, see [DFGS, Figure 15])

The results in Theorem 3.6 may be combined with some observations concerning the conformal invariance of Willmore surfaces. Inverting the solutions found for small $\alpha$ and 
negative $\beta$ yields parametric Willmore surfaces of revolution, which approach the boundary on the left hand side from the left and at the right hand side from the right. In particular they are not generated by graphs.

Concerning nonsymmetric sets of boundary data for $u(-1), u(1), u^{\prime}(-1), u^{\prime}(1)$, existence or nonexistence of solutions to the Dirichlet problem for the Willmore equation are still widely open. Numerical and analytical experiments suggest that such surfaces, which are generated by graphs, may only be found for data which deviate not too much from a symmetric situation. When enlarging the class to parametric surfaces of revolution one may expect a general existence result. Such questions will be subject of future research.

\section{Perspectives: Natural boundary Conditions for Willmore surfaces of} REVOLUTION

Again, we consider surfaces of revolution

$$
\mathcal{R}:[-1,1] \times[0,2 \pi] \ni(x, \varphi) \mapsto(x, u(x) \cos \varphi, u(x) \sin \varphi) \in \mathbb{R}^{3}
$$

generated by graphs of positive smooth functions $u:[-1,1] \rightarrow(0, \infty)$. For given $\alpha>0$ we prescribe the boundary position $u( \pm 1)=\alpha$, but not the boundary slope. Calculating the Euler-Lagrange-equation for the Willmore energy (3.2) within this class of admissible functions now yields an additional boundary term and we find the second condition

$$
H( \pm 1)=0
$$

as a natural boundary condition, see [DG, Lemma A.1]. This means that this setting leads to looking for surfaces of revolution generated by $u$ solving the following Navier boundary value problem:

$$
\left\{\begin{array}{l}
\Delta_{\mathcal{R}} H+2 H\left(H^{2}-K\right)=0 \quad \text { in }(-1,1), \\
u( \pm 1)=\alpha, \quad H( \pm 1)=0 .
\end{array}\right.
$$

An obvious idea is to look for minimal surfaces and indeed, for large enough $\alpha$, one has catenoids as solutions.

Remark 4.1. Let $b_{0}=1.199 \ldots$ be the positive solution of $\cosh \left(b_{0}\right)=b_{0} \sinh \left(b_{0}\right)$ and $\alpha_{0}=$ $\cosh \left(b_{0}\right) / b_{0}=1.508 \ldots$ Then, for $\alpha>\alpha_{0}$, (4.1) has two minimal surface solutions, and for $\alpha=\alpha_{0}$, there is one such solution. These solutions are symmetric with respect to $x=0$.

For small $\alpha>0$, connected minimal surface solutions to (4.1) do not exist, see [DHKW, Chapter 6.1, Theorem 3]. Numerical experiments ([De, Kast]), however, provide evidence that for each $\alpha \in(0, \infty) \backslash\left\{\alpha_{0}\right\}$ one may have at least one further Willmore surface of revolution solving (4.1), which is not a minimal surface. The expected bifurcation diagram is displayed in Figure 13. Apparently, the additional solutions are stable for $\alpha \in\left(0, \alpha_{0}\right)$ and unstable for $\alpha>\alpha_{0}$. The branch of the latter could be tracked numerically until $\alpha \approx 3$.

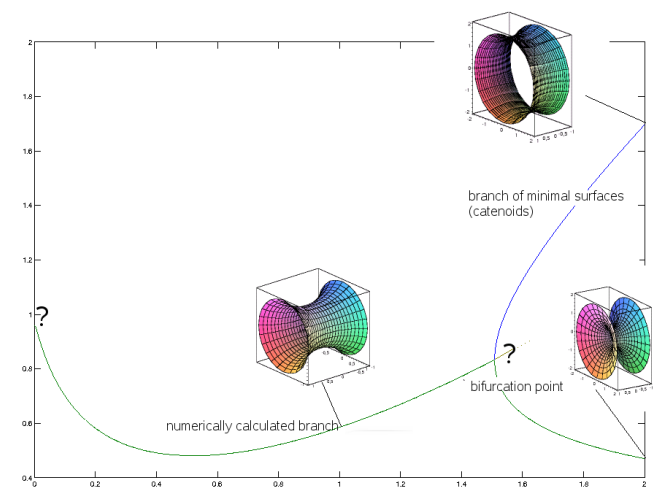

Figure 13. Expected bifurcation diagram (Courtesy of Klaus Deckelnick, see [DG, Figure 1.3]) 
More details concerning (4.1) are discussed in [DG]. A numerical algorithm is proposed and also some analytical facts are proved there. The verification of the full bifurcation diagram (Figure 13) is, however, still open and subject of current research. A possible strategy to find an additional solution could be to show that the solutions for the Dirichlet problem constructed in Theorem 3.6 are unique as minimisers in suitable classes of admissible functions and depend continuously on the data $\alpha, \beta$. Then one could try to find the sign of $H( \pm 1)$ of specific such solutions and try to apply the mean value theorem. Although this strategy looks quite natural it appears to be rather difficult to make it working, at least for $\alpha>\alpha_{0}$.

Boundary value problems for graphs over two-dimensional domains. An important goal will be to understand general boundary value problems for Willmore graphs over twodimensional domains, without imposing symmetry assumptions on the domain and the data. In view of the lack of any general form of comparison principle and the highly nonlinear character of the fourth order Willmore equation, this seems to be a quite difficult task. The previous considerations may be considered as a first step towards developing techniques for future attempts to solve this general problems or at least, significant aspects of it. We hope that the restricted forms of "almost positivity" as discussed in Section 2 may prove to become useful in this respect or may at least explain some features of a possible future theory.

Acknowledgement. I am grateful to my coauthors Anna Dall'Acqua (Magdeburg), Klaus Deckelnick (Magdeburg), Steffen Fröhlich (Berlin), Filippo Gazzola (Milan), Frédéric Robert (Nice), Friedhelm Schieweck (Magdeburg) and Guido Sweers (Cologne) for the very enjoyable collaboration in the articles [DDG, DFGS, DG, GR1, GR2, GS1, GS2, GS3] and the forthcmoing book [GGS] which are basis for the present survey article. I am indebted to Klaus Deckelnick, Friedhelm Schieweck and Guido Sweers for allowing me to use their numerically calculated pictures, see Figures 2, 10, 11, 12 and 13.

Moreover I would like to thank Filippo Gazzola, Carlo Pagani and the "Seminario Matematico Fisico di Milano" for their kind invitation and their hospitality.

\section{REFERENCES}

[ADN] S. Agmon, A. Douglis, L. Nirenberg, Estimates near the boundary for solutions of elliptic partial differential equations satisfying general boundary conditions. I, Commun. Pure Appl. Math. 12 (1959), 623-727.

[BK] M. Bauer, E. Kuwert, Existence of minimizing Willmore surface of prescribed genus, Int. Math. Res. Not. 2003, No.10 (2003), 553-576.

[Bl] S. Blatt, A singular example for the Willmore flow, submitted.

[Bo1] T. Boggio, Sull'equilibrio delle piastre elastiche incastrate, Rend. Acc. Lincei 10 (1901), $197-205$.

[Bo2] T. Boggio, Sulle funzioni di Green d'ordine m, Rend. Circ. Mat. Palermo 20 (1905), 97-135.

[Br] R. Bryant, A duality theorem for Willmore surfaces, J. Diff. Geom. 20 (1984) $23-53$.

[BG] R. Bryant, P. Griffiths, Reduction for constrained variational problems and $\int \frac{1}{2} k^{2} d s$, Amer. J. Math. 108 (1986), 525-570.

[CD] C.V. Coffman, R.J. Duffin, On the structure of biharmonic functions satisfying the clamped condition on a right angle, Adv. Appl. Math., 1 (1980), 373-389.

[CG] C.V. Coffman, C.L. Grover, Obtuse cones in Hilbert spaces and applictions to partial differential equations, J. Funct. Anal. 35 (1980), 369-396.

[C] R. Courant, Dirichlet's Principle, Conformal Mapping, and Minimal Surfaces, Interscience: New York, 1950.

[DA] A. Dall'Acqua, private communication.

[DDG] A. Dall'Acqua, K. Deckelnick, H.-Ch. Grunau, Classical solutions to the Dirichlet problem for Willmore surfaces of revolution, Adv. Calc. Var. 1 (2008), 379-397.

[DFGS] A. Dall'Acqua, S. Fröhlich, H.-Ch. Grunau, F. Schieweck, Symmetric Willmore surfaces of revolution satisfying arbitrary Dirichlet boundary data, Preprint 24-2008, University of Magdeburg, 2008.

[DMS] A. Dall'Acqua, Ch. Meister, G. Sweers, Separating positivity and regularity for fourth order Dirichlet problems in 2d-domains, Analysis 25 (2005), 205-261.

[DS] A. Dall'Acqua, G. Sweers, Estimates for Green function and Poisson kernels of higher-order Dirichlet boundary value problems, J. Differential Equations 205 (2004), 466-487.

[De] K. Deckelnick, private communication. 
[DG] K. Deckelnick, H.-Ch. Grunau, A Navier boundary value problem for Willmore surfaces of revolution, Analysis, Special issue dedicated to Prof. Erhard Heinz on the occasion of his 85th birthday, to appear.

[DHKW] U. Dierkes, S. Hildebrandt, A. Küster, O. Wohlrab, Minimal Surfaces I, Grundlehren der mathematischen Wissenschaften 295, Springer-Verlag: Berlin etc., 1992.

[D1] R.J. Duffin, On a question of Hadamard concerning super-biharmonic functions, J. Math. Phys. 27 (1949), 253-258.

[D2] R.J. Duffin, Continuation of biharmonic functions by reflection, Duke Math. J. 22 (1955), 313-324.

[DKS] G. Dziuk, E. Kuwert, R. Schätzle, Evolution of elastic curves in $\mathbb{R}^{n}$ : Existence and computation, SIAM J. Math. Anal. 33 (2002), 1228-1245.

[Ga] P.R. Garabedian, A partial differential equation arising in conformal mapping, Pacific J. Math. 1 (1951), 485-524.

[GGS] F. Gazzola, H.-Ch. Grunau, G. Sweers, Polyharmonic boundary value problems, Positivity preserving and nonlinear elliptic equations in bounded domains, in preparation.

[GR1] H.-Ch. Grunau, F. Robert, Boundedness of the negative part of biharmonic Green's functions under Dirichlet boundary conditions in general domains, C. R. Math. Acad. Sci. Paris, to appear. H.-Ch. Grunau, F. Robert, Positivity and almost positivity of biharmonic Green's functions under Dirichlet boundary conditions, submitted.

[GS1] H.-Ch. Grunau, G. Sweers, Positivity for perturbations of polyharmonic operators with Dirichlet boundary conditions in two dimensions, Math. Nachr. 179 (1996), 89-102.

[GS2] H.-Ch. Grunau, G. Sweers, Positivity for equations involving polyharmonic operators with Dirichlet boundary conditions, Math. Ann. 307 (1997), 589-626.

[GS3] H.-Ch. Grunau, G. Sweers, Regions of positivity for polyharmonic Green functions in arbitrary domains, Proc. Amer. Math. Society 135 (2007), 3537-3546.

[Ha1] J. Hadamard, Mémoire sur le problème d'analyse relatif à l'équilibre des plaques élastiques encastrées, in: Euvres de Jacques Hadamard, Tome II, 515-641, CNRS: Paris 1968.

[Ha2] J. Hadamard, Sur certains cas intéressants du problème biharmonique, in: Euvres de Jacques Hadamard, Tome III, 1297-1299, CNRS: Paris 1968.

[HJS] H. Hedenmalm, S. Jakobsson, S. Shimorin, A biharmonic maximum principle for hyperbolic surfaces, J. Reine Angew. Math. 550 (2002), 25-75.

[He] W. Helfrich, Elastic properties of lipid bylayers: Theory and possible experiments, Z. Naturforsch. C 28 (1973), 693-703.

[HP] U. Hertrich-Jeromin, U. Pinkall, Ein Beweis der Willmoreschen Vermutung für Kanaltori, J. Reine Angew. Math. 430 (1992), 21-34.

[Kast] D. Kastsian, Finite element approximation of two-dimensional rotationally symmetric Willmore surfaces, Master's thesis, University of Magdeburg, 2007.

[KaSw] B. Kawohl, G.Sweers, On 'anti'-eigenvalues for elliptic systems and a question of McKenna and Walter, Indiana Univ. Math. J. 51 (2002), 1023-1040.

$[\mathrm{KKM}]$ V.A. Kozlov, V.A. Kondrat'ev, V.G. Maz'ya, On sign variation and the absence of "strong" zeros of solutions of elliptic equations, Math. USSR Izvestiya 34 (1990), 337-353.

[Kr1] Ju.P. Krasovski1, Investigation of potentials connected with boundary value problems for elliptic equations, Izv. Akad. Nauk SSSR Ser. Mat. 31 (1967), 587-640, English translation in: Math. USSR, Izv. 1 (1967), 569-622.

[Kr2] Ju.P. Krasovskiǔ, Isolation of singularities of the Green's function (Russian), Izv. Akad. Nauk SSSR Ser. Mat. 31 (1967), 977-1010, English translation in: Math. USSR, Izv. 1 (1967), 935-966.

[KuS1] E. Kuwert, R. Schätzle, The Willmore flow with small initial energy, J. Differ. Geom. 57 (2001), 409-441.

[KuS2] E. Kuwert, R. Schätzle, Gradient flow for the Willmore functional, Commun. Anal. Geom. 10 (2002), 307-339.

[KuS3] E. Kuwert, R. Schätzle, Removability of point singularities of Willmore surfaces, Annals of Math. 160 (2004), 315-357.

[KuS4] E. Kuwert, R. Schätzle, Estimation of the conformal factor under bounded Willmore energy modulo the Möbius group, Preprint, 2008.

[KuS5] E. Kuwert, R. Schätzle, Minimizers of the Willmore functional under fixed conformal class, Preprint, 2008

[LS1] J. Langer, D. Singer, The total squared curvature of closed curves, J. Differ. Geom. 20 (1984), $1-22$.

[LS2] J. Langer, D. Singer, Curves in the hyperbolic plane and mean curvature of tori in 3-space, Bull. London Math. Soc. 16 (1984), 531-534.

[LP] K. Leschke, F. Pedit, Sequences of Willmore surfaces, Math. Z. 259 (2008), 113-122.

[L] Ch. Loewner, On generation of solutions of the biharmonic equation in the plane by conformal mappings, Pacific J. Math. 3 (1953), 417-436.

[MSt] U.F. Mayer, G. Simonett, A numerical scheme for axisymmetric solutions of curvature-driven free boundary problems, with applications to the Willmore flow, Interfaces Free Bound. 4 (2002), 89-109. 
[MSh] V.G. Maz'ya, T.O. Shaposhnikova, Jacques Hadamard, A universal mathematician, History of Mathematics 14, American Mathematical Society: Providence (RI), London Mathematical Society: London, 1998.

[Ne] Z. Nehari, On the biharmonic Green's function, in: Studies Math. Mech., presented to Richard von Mises, pp. 111-117, Academic Press: New York etc. 1954.

[Nic] M. Nicolesco, Les fonctions polyharmoniques, Hermann: Paris 1936.

[Nit] J.C.C. Nitsche, Boundary value problems for variational integrals involving surface curvatures. Quart. Appl. Math. 51, 363-387, 1993.

[O] St. Osher, On Green's function for the biharmonic equation in a right angle wedge, J. Math. Anal. Appl. 43 (1973), 705-716.

[Poi1] S.D. Poisson, Mémoire sur les surfaces élastiques, Cl. Sci. Mathém. Phys. Inst. de France, 2nd pt, (1812), 167-225.

[Poi2] S.D. Poisson, Mémoire sur le calcul des variations, Mém. Acad. Royal Sci. Inst. de France 12 (1833), 223-331.

[Pol] A. Polden, Curves and Surfaces of Least Total Curvature and Fourth-Order Flows, Ph.D. dissertation, University of Tübingen, 1996

[R] T. Rivière, Analysis aspects of Willmore surfaces, Invent. Math. 174 (2008), 1-45.

[Sa] E. Sassone, Positivity for polyharmonic problems on domains close to a disk, Ann. Mat. Pura Appl. 186 (2007), 419-432.

[SchR] R. Schätzle, The Willmore boundary value problem, Preprint, 2006.

[SchM] M. Schmidt, Existence of minimizing Willmore surfaces of prescribed conformal class, Preprint, arXiv:math/0403301v1, 2004.

[SchJ] J. Schröder, Operator inequalities, Math. Science Engineering 147, Academic Press: New York etc. 1980.

[Se] J.B. Seif, On the Green's function for the biharmonic equation in an infinite wedge, Trans. Amer. Math. Soc. 182 (1973), 241-260.

[ShT] H.S. Shapiro, M. Tegmark, An elementary proof that the biharmonic Green function of an eccentric ellipse changes sign, SIAM Rev. 36 (1994), 99-101.

[Sn] L. Simon, Existence of surfaces minimizing the Willmore functional, Commun. Anal. Geom. 1 (1993), 281-326.

[St] G. Simonett, The Willmore flow near spheres, Differ. Integral Equ. 14 (2001), 1005-1014.

[Th] G. Thomsen, Über konforme Geometrie I: Grundlagen der konformen Flächentheorie, Abh. Math. Sem. Hamburg 3 (1923), 31-56.

[Wi] T.J. Willmore, Riemannian geometry, Oxford Science Publications, Clarendon Press, 1993.

Fakultät für Mathematik, Otto-von-Guericke-Universität, Postfach 4120, D-39016 MagdeBURG (GERMANY)

E-mail address: Hans-Christoph.Grunau@ovgu.de 